\title{
Using Delayed Feedback for Antenna Selection in MIMO Systems
}

\author{
T. R. Ramya and Srikrishna Bhashyam
}

\begin{abstract}
Antenna selection in Multiple-Input-Multipleoutput (MIMO) systems preserves diversity gain while significantly reducing hardware complexity. However, imperfect Channel State Information (CSI) affects performance. In this paper, we first analyze the performance of a MIMO system employing antenna selection at the transmitter and Maximal Ratio Combining (MRC) at the receiver in the presence of feedback delay and channel estimation errors. Then, we determine whether channel prediction can compensate for the effect of feedback delay. Outage probability is analyzed as a function of $\rho$, the correlation coefficient between the CSI used at the receiver for decoding (CSIR) and the CSI used at the transmitter for selection (CSIT). Analytical results show that the effect of feedback delay is more significant than the effect of estimation error. In order to overcome the effect of delay, $\rho$ should increase with SNR. For a given SNR, the length of the Linear Minimum Mean Square Error (LMMSE) prediction filter required is calculated and shown to increase with SNR. Finally, we determine the asymptotic diversity order as a function of the feedback quality. Results show that if $1-\rho \propto S N R^{-1}$, the diversity order with imperfect CSI is same as that with perfect CSI.
\end{abstract}

Index Terms-Antenna selection, feedback delay, channel prediction, diversity.

\section{INTRODUCTION}

$\mathbf{I}$ NFORMATION theoretic studies have shown that employing multiple antennas at the transmitter and/or at the receiver leads to improvement in the capacity and outage performance of wireless communication systems [1]. Further improvement in performance can be achieved when Channel State Information (CSI) is used at the transmitter to perform adaptation [2], [3]. However, adaptive multi-antenna systems suffer from two major bottlenecks. The first bottleneck is the increase in hardware complexity due to the requirement of an additional radio frequency (RF) chain for each additional antenna. The second bottleneck is the necessity of CSI at the transmitter (CSIT). In practice, perfect CSIT is not possible due to channel estimation errors, feedback delay, and feedback channel errors.

Transmit antenna selection, where a large number of antennas are employed and only the best subset is used for transmission, is a promising multi-antenna technique as: (1) significant reduction in hardware complexity can be achieved since only

Manuscript received March 2, 2009; revised June 3, 2009 and August 13, 2009; accepted August 13, 2009. The associate editor coordinating the review of this paper and approving it for publication was N. Mehta.

The authors are with the Department of Electrical Engineering, Indian Institute of Technology Madras, Chennai, India (e-mail: skrishna@ee.iitm.ac.in).

This work was presented in part at the National Conference on Communications held at Mumbai, India in February 2008. This work was supported in part by the Department of Science and Technology, India.

Digital Object Identifier 10.1109/TWC.2009.12.090304 a subset of antennas are simultaneously used, (2) diversity gain is unaltered, because it is the number of total available antennas that determines the diversity gain, irrespective of the number of antennas that are used simultaneously [4], (3) lower feedback bandwidth is required (only the selected index needs to be fed back) and (4) low feedback rate is sufficient since the rate of variation of the maximum index is lower compared to the rate of channel variation.

The outage performance of the Multiple Input Multiple Output (MIMO) transmit antenna selection scheme with maximal ratio combining (MRC) at the receiver is presented in [5] and the outage probability of the transmit antenna subset selection system employing space time coding is presented in [6]. It has been established in [5] and [6] that when CSIT is perfect, antenna selection preserves the diversity gain. However, imperfect CSI affects the selection process as well as the decoding process. In [7], the author has shown that the diversity order of the single antenna selection system depends on the ordinal number of the selected antenna. Therefore, it is evident that wrong selection degrades the diversity order and thereby affects the performance. The effect of channel estimation errors on transmit antenna selection systems have been extensively studied in [8]-[12]. Simulation results on the loss in the achievable capacity of the hybrid selection MRC system due to CSI imperfections is presented in [8]. The error probability of Generalized Selection Combining is presented in [9], where it was shown that estimation errors lead to a bounded loss in Signal to Noise Ratio (SNR) compared to the perfect CSI case. The effect of channel estimation error on a MIMO OFDM system employing antenna selection is analyzed in [10]. In [11], the SER of the antenna subset selection system is studied in the presence of channel estimation errors. The effect of channel estimation errors on the system employing joint transmit and receive antenna selection along with space time coding is studied in [12]. It has been established in [8]-[12] that estimation errors lead to a bounded SNR loss and do not affect the achievable diversity gain.

The effect of feedback delay on the error rate of a system employing Alamouti Space Time Block Coding (STBC) with antenna selection and M-QAM constellations is studied in [13]. Similarly, the effect of feedback delay on MIMO systems employing rate adaptation has been presented in [14]. Both [13] and [14] study the uncoded Symbol Error rate (SER) performance for QAM constellations, and neglect channel estimation error at the receiver. In [15], the SER and outage probability of transmit antenna selection in the presence of feedback delay are derived for the case of single receive antenna and perfect CSI at the receiver. Both feedback delay and 
channel estimation errors are considered in [16] and [17] for an antenna selection system for some specific scenarios. While [16] presents only an approximate bit error rate expression for BPSK modulation for very small and very large feedback delays, [17] considers only a $2 \times 1$ MIMO system using BPSK or QPSK. Furthermore, the use of prediction to compensate for the effect of feedback delay has not been considered in [13]-[17].

In this paper, we analyze the performance of a Multiple Input Multiple Output (MIMO) system employing transmit antenna selection and Maximal Ratio combining (MRC) at the receiver in the presence of feedback delay and channel estimation errors. A generalization of the imperfect CSIT model in [18] is used, where the effect of mismatched CSI between the transmitter and receiver is captured using $\rho$, the correlation coefficient between the CSI used for antenna selection at the transmitter (CSIT) and the CSI available at the receiver (CSIR) for decoding. The outage probability of the above system is analyzed as a function of $\rho$. The outage capacity is also analyzed to study effect of imperfect CSI on a rate adaptive system. The value of $\rho$ required to limit degradation is calculated and found to increase with SNR. Channel prediction is required to achieve the required $\rho$ at medium and high SNR. The length of the prediction filter so required is also calculated numerically. The asymptotic diversity order is also analyzed as a function the feedback quality $\alpha$, defined as the rate at which $\rho$ approaches one as the Signal to Noise Ratio (SNR) tends to infinity. Analytical results reveal that, when $\alpha=1$, diversity order is the same as with perfect CSIT.

The effect of delayed feedback on Eigen beamforming for MIMO systems has been presented in [19], while the effect of feedback delay on beamforming in MISO systems has been presented in [18]. Feedback channel errors are not considered in our paper. We assume that the index of the selected antenna is received without error after a delay. The effect of errors in the feedback channel have been considered in [20]. In [20], when errors occur in the feedback, the transmitter selects an antenna different from the one signaled by the receiver leading to the receiver using the wrong channel estimate for decoding.

The rest of the paper is organized as follows: Section II describes the system model. Section III presents the analysis of the outage probability of MIMO antenna selection system with imperfect CSIT. Numerical results on the value of $\rho$ required to limit degradation are also presented in Section III. Analysis of the asymptotic diversity order is presented in Section IV and conclusions are drawn in Section V.

\section{System Model}

The system model is depicted in Fig 1. A MIMO system with $N_{t}$ transmit antennas and $N_{r}$ receive antennas is considered. At any instant of time, one out of the $N_{t}$ antennas is used for transmission. The channel between the transmitter and receiver is assumed to be frequency flat. The received vector at time index $k$ is therefore, represented as:

$$
\mathbf{y}(k)=\sqrt{P} \mathbf{h}_{\text {sel }}(k) x(k)+\mathbf{n}(k),
$$

where $x(k)$ represents the transmit symbol at time $k, P$ is the transmit power, $\mathbf{n}(k) \sim \mathcal{C N}\left(0, \sigma_{n}^{2} \mathbf{I}_{N_{r}}\right)$ is the vector of

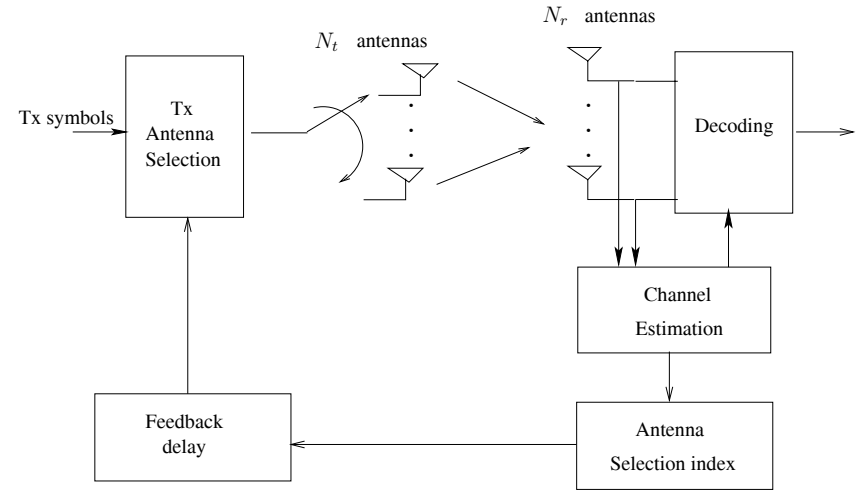

Fig. 1. System model.

Additive White Gaussian Noise (AWGN) and $\mathbf{h}_{\text {sel }}(k)$ is the $N_{r} \times 1$ channel vector corresponding to the selected transmit antenna. $\mathbf{h}_{s e l}(k)$ is one of the columns of the $N_{r} \times N_{t}$ channel matrix $\mathbf{H}(k)$ at instant $k$. The elements of $\mathbf{H}(k)$ are assumed to be i.i.d and $\mathcal{C N}(0,1)$. A block fading model is considered, where the elements of $\mathbf{H}$ are assumed to be constant over a block and correlated across blocks.

MRC is employed at the receiver for decoding. CSI is estimated using training symbols. Antenna selection is done based on the estimated CSI and the selected index is fed back to the transmitter. Selection is based on maximizing the instantaneous receive SNR, i.e., the antenna that maximizes $\left\|\mathbf{h}_{\text {sel }}(k)\right\|^{2}$ is selected. Due to feedback delay, switching is effective only a few blocks after selection. Therefore, antenna selection at any instant is based on the old CSI estimate. The effect of feedback delay can be significantly reduced using channel prediction. Since the channel exhibits temporal correlation, future CSI can be predicted at the receiver using the present and past CSI, and used for antenna selection.

\section{A. Imperfect CSI Model}

CSI is estimated using training symbols. The training pattern employed for channel estimation is similar to the training in [21], [22] and is also depicted in Fig. 2. At the beginning of every block, the first $N_{t}$ symbols are assigned for training, one for each transmit antenna. Therefore, channel is estimated once in every $T$ seconds, where $T$ is the frame duration. Minimum mean-squared error (MMSE) channel estimation is assumed. The training symbol power can be increased compared to the data symbol power, without changing the average transmit power, to improve the quality of the estimate [21][22]. Assuming that the number of symbols in the frame is much larger compared to $N_{t}$, increasing the training power leads to a negligible change in the data power in order to maintain the same average transmit power. Let $\mathbf{H}_{t}$ represent the channel estimate used for antenna selection and $\mathbf{H}_{r}$ represent the channel estimate used for decoding. Since $\mathbf{H}_{t}$ and $\mathbf{H}_{r}$ are both zero mean and jointly Gaussian and circularly symmetric, they can be related as follows:

$$
\mathbf{H}_{t}=\sigma_{t}\left[\frac{\rho}{\sigma_{r}} \mathbf{H}_{r}+\sqrt{1-\rho^{2}} \mathbf{E}\right],
$$




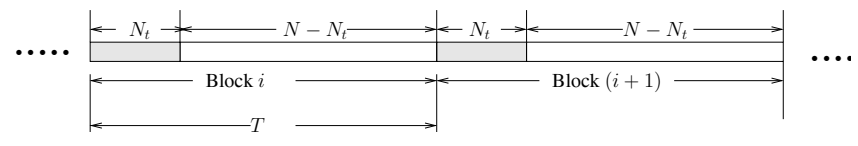

Fig. 2. Training model.

where $E_{i j} \sim \mathcal{C N}(0,1), \sigma_{r}^{2}=E\left[\left|H_{r, i j}\right|^{2}\right], \sigma_{t}^{2}=E\left[\left|H_{t, i j}\right|^{2}\right]$, and $\rho=\frac{E\left[H_{t, i j} H_{r, i j}^{*}\right]}{\sqrt{E\left[\left|H_{r, i j}\right|^{2}\right] E\left[\left|H_{t, i j}\right|^{2}\right]}} . X_{i j}$ represents the $(i, j)^{t h}$ element of the matrix $\mathbf{X}$. Since $H_{t, i j}$ 's are i.i.d and $H_{r, i j}$ 's are also i.i.d., $\rho$ is independent of $i, j$. Note that $\mathbf{H}_{t}$ and $\mathbf{H}_{r}$ can also be related as follows:

$$
\mathbf{H}_{r}=\sigma_{r}\left[\frac{\rho}{\sigma_{t}} \mathbf{H}_{t}+\sqrt{1-\rho^{2}} \mathbf{E}_{1}\right],
$$

where $E_{1, i j} \sim \mathcal{C N}(0,1)$, and $\sigma_{r}^{2}, \sigma_{t}^{2}$, and $\rho$ are as defined above. Note that $\mathbf{E}_{1}$ in equation (3) and $\mathbf{E}$ in equation (2) are different even though they have the same statistical properties. The following CSI imperfections are important special cases considered in existing work and in comparisons in later sections.

\section{Case 1: Estimation errors, No feedback delay}

In this case, $\mathbf{H}_{t}=\mathbf{H}_{r}=$ estimated CSI, $\hat{\mathbf{H}}$.

$\rho=1$ and $\sigma_{t}^{2}=\sigma_{r}^{2}=\left(1-\sigma_{e}^{2}\right)=\frac{P_{t}}{P_{t}+\sigma_{n}^{2}}$,

where $\sigma_{e}^{2}$ is the estimation error variance and $P_{t}$ is the transmit training power.

\section{Case 2: Feedback delay, No estimation error} In this case, $\mathbf{H}_{t}=$ past channel $\mathbf{H}_{\text {old }}$ and $\mathbf{H}_{r}=\mathbf{H}$. $\sigma_{t}^{2}=\sigma_{r}^{2}=1$ and $\rho=J_{0}\left(2 \pi f_{d} T \Delta\right)$,

where $J_{0}(x)=$ zeroth order Bessel function, $f_{d}=$ Doppler frequency, and $\Delta=$ feedback delay in frames. It is clear that $\rho$ is independent of SNR and is strictly less than 1 as $S N R \rightarrow \infty$.

Case 3: Estimation errors + Feedback delay

In this case, $\mathbf{H}_{t}=\hat{\mathbf{H}}_{\text {old }}$ and $\mathbf{H}_{r}=\hat{\mathbf{H}}$.

$\rho=\frac{P_{t}}{P_{t}+\sigma_{n}^{2}} J_{0}\left(2 \pi f_{d} T \Delta\right)$.

As $S N R \rightarrow \infty, \frac{P_{t}}{P_{t}+\sigma_{n}^{2}} \rightarrow 1$ and $\rho \rightarrow J_{0}\left(2 \pi f_{d} T \Delta\right)<1$.

Case 4: Estimation errors + Feedback delay + Channel prediction

In this case, $\mathbf{H}_{r}=\hat{\mathbf{H}}$, and $\mathbf{H}_{t}=\tilde{\mathbf{H}}$, where $\tilde{\mathbf{H}}$ is the predicted CSI. We have considered an $L$-tap linear MMSE prediction filter [23] to predict the true channel value using the past channel estimates. Let $\tilde{H}_{i j}(k)=\mathbf{w}^{H} \mathbf{h}_{i j, \Delta}(k)$, where $\mathbf{h}_{i j, \Delta}(k)=\left[\hat{H}_{i j}(k-\Delta), \hat{H}_{i j}(k-\Delta-1), \cdots, \hat{H}_{i j}(k-\Delta-\right.$ $L+1)]$, and $\mathbf{w}$ is the vector of prediction filter coefficients. At time $k, \tilde{H}_{i j}(k+\Delta)$ is computed and used for antenna selection. Since the channel between different pairs of transmit and receive antennas are i.i.d., the linear MMSE prediction filter coefficients are independent of $i$ and $j$ and given by [23]

$$
\mathbf{w}=\mathbf{R}^{-1} \mathbf{p},
$$

where $\mathbf{R}=E\left[\mathbf{h}_{i j, \Delta}(k) \mathbf{h}_{i j, \Delta}(k)^{H}\right]$ and $\mathbf{p}=$ $E\left[H_{i j}(k) \mathbf{h}_{i j, \Delta}(k)^{H}\right]$. The $i^{t h}$ element of vector $\mathbf{p}$ is given by

$$
p_{i}=\frac{P_{t}}{P_{t}+\sigma_{n}^{2}} J_{0}\left(2 \pi f_{d} T(\Delta+i-1)\right),
$$

and the elements of the matrix $\mathbf{R}$ are given by

$$
\begin{aligned}
& R_{i j}=\left(\frac{P_{t}}{P_{t}+\sigma_{n}^{2}}\right) \\
& {\left[\frac{P_{t}}{P_{t}+\sigma_{n}^{2}} J_{0}\left(2 \pi f_{d} T(j-i)\right)+\frac{\sigma_{n}^{2}}{P_{t}+\sigma_{n}^{2}} \delta(i-j)\right],}
\end{aligned}
$$

where $\delta(i-j)$ is 1 if $i=j$ and 0 if $i \neq j$. Further, it can be shown that

$$
\sigma_{t}^{2}=\mathbf{p}^{H} \mathbf{w}=\mathbf{p}^{H} \mathbf{R}^{-1} \mathbf{p}
$$

and

$$
\rho=\sqrt{\frac{P_{t}}{P_{t}+\sigma_{n}^{2}} \mathbf{p}^{H} \mathbf{R}^{-1} \mathbf{p} .}
$$

Predicting future channel values improves the performance of adaptive transmission systems [25]. It has been shown that a band-limited process can be predicted exactly using past samples with an infinite order predictor in the absence of channel estimation errors (i.e. $\rho$ can be 1) [24]. This is not possible with a finite-tap predictor and $\rho$ is strictly less than 1 [24] even without channel estimation errors. However, $\rho$ increases with increasing filter order [23], [25], [26]. In the presence of channel estimation errors, a continuous-time prediction filter observing the channel over a finite duration interval of past values can achieve $\rho \rightarrow 1$ as SNR tends to infinity [27]. This continuous-time prediction filter provides a performance bound for other discrete-time predictors based on samples.

Knowledge of Doppler spread is assumed in the design of the above prediction filter. In practice, the Doppler spread has to be estimated. Methods for Doppler spread estimation in Rayleigh fading channels are presented in [28], and, more specifically, in the context of OFDM systems in [29], [30].

\section{Outage Analysis of Mimo Antenna Selection WITH IMPERFECT CSI}

The index of the selected antenna is determined using $\mathbf{H}_{t}$ and fed back to the transmitter through a feedback channel with delay. The channel estimate $\mathbf{H}_{r}$ is obtained from the training at the beginning of the block for decoding. Both $\mathbf{H}_{t}$ are $\mathbf{H}_{r}$ are known to the receiver. Therefore, the outage probability is:

$$
P(\text { outage })=P\left(I\left(x ; \mathbf{y} / \mathbf{H}_{t}, \mathbf{H}_{r}\right)<R\right),
$$

where $R$ is the required rate. The mutual information between $x$ and $\mathbf{y}$ of the MIMO antenna selection system in the presence of imperfect CSIT can be lower bounded as follows [21], [22]:

$$
I\left(x ; \mathbf{y} / \mathbf{H}_{t}, \mathbf{H}_{r}\right) \geq \log \left(1+\Gamma|| \mathbf{H}_{r, s e l} \|^{2}\right),
$$

where $\Gamma=\frac{P_{d}}{P_{d} \sigma_{e}^{2}+\sigma_{n}^{2}}, P_{d}$ is the transmit data power and $\mathbf{H}_{r, s e l}$ is the estimate of the channel vector corresponding to the selected antenna. In the absence of channel estimation errors, i.e., perfect CSIR, equation (5) becomes an equality. Using 
equation (5), we can get an upper bound on outage probability as follows:

$$
P(\text { outage }) \leq P\left(\left\|\mathbf{H}_{r, \text { sel }}\right\|^{2}<\frac{e^{R}-1}{\Gamma}\right) .
$$

Selection is based on $\mathbf{H}_{t}$, i.e., the selected antenna index is equal to the $\arg \max _{i=1,2, \cdots, N_{t}}\left\|\mathbf{H}_{t, i}\right\|^{2}$, where $\mathbf{H}_{t, i}$ is the $i^{\text {th }}$ column of $\mathbf{H}_{t}$. Therefore, we have

$$
\begin{gathered}
P(\text { outage }) \leq \sum_{i=1}^{N_{t}} P\left(\left\|\mathbf{H}_{r, i}\right\|^{2}<\beta, \text { Antenna } i \text { is selected }\right) \\
=\sum_{i=1}^{N_{t}} P\left(\left\|\mathbf{H}_{r, i}\right\|^{2}<\beta,\left\|\mathbf{H}_{t, i}\right\|^{2}=\max _{j=1,2, \cdots, N_{t}}\left\|\mathbf{H}_{t, j}\right\|^{2}\right),
\end{gathered}
$$

where $\beta=\frac{e^{R}-1}{\Gamma}$. From equation (3), we can write

$$
\begin{gathered}
\left\|\mathbf{H}_{r, i}\right\|^{2}=\frac{\sigma_{r}^{2}\left(1-\rho^{2}\right)}{2}\left\|\left[\sqrt{\frac{2 \rho^{2}}{\left(1-\rho^{2}\right) \sigma_{t}^{2}}} \mathbf{H}_{t, i}+\sqrt{2} \mathbf{E}_{1, i}\right]\right\|^{2} \\
=\sigma_{r}^{2} \frac{\left(1-\rho^{2}\right)}{2} A_{i},
\end{gathered}
$$

where $A_{i}$ is defined as $\left\|\left[\sqrt{\frac{2 \rho^{2}}{\left(1-\rho^{2}\right) \sigma_{t}^{2}}} \mathbf{H}_{t, i}+\sqrt{2} \mathbf{E}_{1, i}\right]\right\|^{2}$. Define $Z_{i}=\left\|\mathbf{H}_{t, i}\right\|^{2}, Z=\max _{j=1,2, \cdots, N_{t}} Z_{j}$, and $\mu=\frac{\rho^{2}}{1-\rho^{2}}$. Now, $\frac{1}{1-\rho^{2}}=1+\mu$, and the outage probability bound can be rewritten as

$$
P(\text { outage }) \leq \sum_{i=1}^{N_{t}} P\left(A_{i}<2 \beta\left(\frac{1+\mu}{\sigma_{r}^{2}}\right), Z=Z_{i}\right) .
$$

Let $\nu=\beta\left(\frac{1+\mu}{\sigma_{r}^{2}}\right)$. Since $A_{i}$,s are i.i.d and $Z_{i}$ 's are also i.i.d., by symmetry we get

$$
\begin{aligned}
P(\text { outage }) & \leq N_{t} P\left(A_{1}<2 \nu, Z=Z_{1}\right) \\
& =N_{t} P\left(A_{1}<2 \nu / Z=Z_{1}\right) P\left(Z=Z_{1}\right) \\
& =P\left(A_{1}<2 \nu / Z=Z_{1}\right) \\
& =\int_{0}^{\infty} P\left(A_{1}<2 \nu / Z=Z_{1}=z\right) f_{Z / Z=Z_{1}}(z) d z \\
& =\int_{0}^{\infty} P\left(A_{1}<2 \nu / Z=Z_{1}=z\right) f_{Z}(z) d z . \quad(10)
\end{aligned}
$$

Note that, by symmetry, $P\left(Z=Z_{1}\right)=1 / N_{t}$ and $f_{Z / Z=Z_{1}}(z)=f_{Z}(z)$. Since the elements of $\sqrt{2} \mathbf{E}_{1, i}$ are complex Gaussian with variance 1 per dimension, for a given $\mathbf{H}_{t, i}$, $A_{i}$ is non-central distributed with $2 N_{r}$ degrees of freedom. The non centrality parameter $\delta_{i}=\frac{2 \mu}{\sigma_{t}^{2}}|| \mathbf{H}_{t, i} \|^{2}$. Therefore, we have

$$
P\left(A_{1}<2 \nu / Z=Z_{1}=z\right)=F_{n c-\chi^{2}, 2 N_{r}, \delta_{1}}(2 \nu),
$$

where $F_{n c-\chi^{2}, 2 N_{r}, \delta_{1}}(\cdot)$ is the CDF of the non central chisquared distributed random variable with parameters $2 N_{r}$ and $\delta_{1}$ given by (equation (26.4.25) in [31])

$$
F_{n c-\chi^{2}, 2 N_{r}, \delta_{1}}(a)=\sum_{j=0}^{\infty} \frac{e^{-\frac{\delta_{1}}{2}}\left(\frac{\delta_{1}}{2}\right)^{j}}{j !} \gamma_{j+N_{r}}\left(\frac{a}{2}\right)
$$

where $\gamma_{i}(x)$ is the regularized gamma function given by $\gamma_{i}(x)=\int_{0}^{x} \frac{e^{-y} y^{i-1}}{(i-1) !} d y$. Therefore, we have

$$
P(\text { outage }) \leq \int_{0}^{\infty} F_{n c-\chi^{2}, 2 N_{r}, \delta}(2 \nu) f_{Z}(z) d z .
$$

Now, we find $f_{Z}(z)$. Each $Z_{j}$ is sum of squared magnitudes of $N_{r}$ zero-mean complex Gaussian random variables with variance $\frac{\sigma_{t}^{2}}{2}$ per dimension. Therefore, $Z_{j}$ is chi-squared distributed with $\operatorname{PDF} f_{Z_{j}}(z)=\frac{e^{-\frac{z}{\sigma_{t}^{2}}\left(\frac{z}{\sigma_{t}^{2}}\right)^{N_{r}-1}}}{\sigma_{t}^{2}\left(N_{r}-1\right) !}$ and $\mathrm{CDF}$ $F_{Z_{j}}(z)=\gamma_{N_{r}}\left(\frac{z}{\sigma_{t}^{2}}\right)$. Since $Z$ is maximum of $N_{t}$ i.i.d. random variables, the pdf of $Z$ is given by

$$
\begin{aligned}
f_{Z}(z) & =N_{t}\left[F_{Z_{j}}(z)\right]^{N_{t}-1} f_{Z_{j}}(z) \\
& =N_{t}\left[\gamma_{N_{r}}\left(\frac{z}{\sigma_{t}^{2}}\right)\right]^{N_{t}-1} \frac{e^{-\frac{z}{\sigma_{t}^{2}}\left(\frac{z}{\sigma_{t}^{2}}\right)^{N_{r}-1}}}{\sigma_{t}^{2}\left(N_{r}-1\right) !} .
\end{aligned}
$$

Since $\gamma_{N_{r}}(x)=1-e^{-x} \sum_{i=0}^{N_{r}-1} \frac{x^{i}}{i !}$, powers of $\gamma_{N_{r}}(x)$ can be found using binomial and multinomial expansion (equation (24.1.2B) in [31]). Therefore, we have

$$
\begin{aligned}
{\left[\gamma_{N_{r}}\left(\frac{z}{\sigma_{t}^{2}}\right)\right]^{N_{t}-1}=} & \sum_{l=0}^{N_{t}-1}\left(\begin{array}{c}
N_{t}-1 \\
l
\end{array}\right)(-1)^{l} e^{-\frac{l z}{\sigma_{t}^{2}}} \\
& \sum_{b_{1}, b_{2}, \cdots, b_{N_{r}}}\left(\begin{array}{c}
l \\
b_{1}, b_{2}, \cdots, b_{N_{r}}
\end{array}\right) \frac{\left(\frac{z}{\sigma_{t}^{2}}\right)^{M}}{L_{1}}
\end{aligned}
$$

where the integer vector $\mathbf{b}=\left[b_{1}, b_{2}, \cdots, b_{N_{r}}\right]$ is such that $\sum_{t=1}^{N_{r}} b_{t}=l, L_{1}=\Pi_{t=0}^{N_{r}-1}(t !)^{b_{t+1}}$ and $M=\sum_{t=0}^{N_{r}-1} t b_{t+1}$. Using equation (14) in equation(13), we get

$$
\begin{aligned}
& f_{Z}(z)=N_{t} \sum_{l=0}^{N_{t}-1}\left(\begin{array}{c}
N_{t}-1 \\
l
\end{array}\right)(-1)^{l} \\
& \sum_{b_{1}, b_{2}, \cdots, b_{N_{r}}}\left(b_{1}, b_{2}, \cdots, b_{N_{r}}\right) \frac{1}{L_{1}} \frac{e^{-\frac{z(1+l)}{\sigma_{t}^{2}}}\left(\frac{z}{\sigma_{t}^{2}}\right)^{M+N_{r}-1}}{\sigma_{t}^{2}\left(N_{r}-1\right) !} \\
& =N_{t} \sum_{l=0}^{N_{t}-1} \sum_{\mathbf{b}} C_{l, \mathbf{b}} \frac{e^{-\frac{z(1+l)}{\sigma_{t}^{2}}}\left(\frac{z}{\sigma_{t}^{2}}\right)^{M+N_{r}-1}}{\sigma_{t}^{2}\left(N_{r}-1\right) !}
\end{aligned}
$$

where

$$
C_{l, \mathbf{b}}=\left(\begin{array}{c}
N_{t}-1 \\
l
\end{array}\right)(-1)^{l}\left(\begin{array}{c}
l \\
b_{1}, b_{2}, \cdots, b_{N_{r}}
\end{array}\right) \frac{1}{L_{1}}
$$

The expressions in equations (16) and (11) can be substituted in equation (12) to get:

$$
\begin{aligned}
& P(\text { outage }) \leq N_{t} \sum_{l=0}^{N_{t}-1} \sum_{\mathbf{b}} C_{l, \mathbf{b}} \sum_{j=0}^{\infty} \frac{\mu^{j}}{j !} \gamma_{j+N_{r}}(\nu) \\
& \int_{0}^{\infty} \frac{e^{-\frac{z(1+l+\mu)}{\sigma_{t}^{2}}}\left(\frac{z}{\sigma_{t}^{2}}\right)^{j+M+N_{r}-1}}{\sigma_{t}^{2}\left(N_{r}-1\right) !} d z
\end{aligned}
$$




$$
\begin{aligned}
& =N_{t} \sum_{l=0}^{N_{t}-1} \sum_{\mathbf{b}} C_{l, \mathbf{b}}\left(\frac{1}{1+l+\mu}\right)^{M+N_{r}} \sum_{j=0}^{\infty}\left(\frac{\mu}{1+l+\mu}\right)^{j} \\
& \frac{\left(j+M+N_{r}-1\right) !}{j !\left(N_{r}-1\right) !} \gamma_{j+N_{r}}(\nu) \\
& =N_{t} \sum_{l=0}^{N_{t}-1} \sum_{\mathbf{b}} C_{l, \mathbf{b}}\left(\frac{1}{1+l+\mu}\right)^{M+N_{r}} \sum_{j=0}^{\infty}\left(\frac{\mu}{1+l+\mu}\right)^{j} \\
& \frac{\left(j+M+N_{r}-1\right) !}{j !\left(N_{r}-1\right) !}\left[\int_{0}^{\nu} \frac{e^{-a} a^{j+N_{r}-1}}{\left(j+N_{r}-1\right) !} d a\right] \\
& =N_{t} \sum_{l=0}^{N_{t}-1} \sum_{\mathbf{b}} C_{l, \mathbf{b}}\left(\frac{1}{1+l+\mu}\right)^{M+N_{r}} \int_{0}^{\nu} \frac{e^{-a} a^{N_{r}-1}}{\left(N_{r}-1\right) !} \\
& \sum_{j=0}^{\infty}\left(\frac{a \mu}{1+l+\mu}\right)^{j} \frac{\left(j+M+N_{r}-1\right) !}{j !\left(j+N_{r}-1\right) !} d a \\
& =N_{t} \sum_{l=0}^{N_{t}-1} \sum_{\mathbf{b}} C_{l, \mathbf{b}} \frac{\left(N_{r}+M-1\right) !}{\left(N_{r}-1\right) !}\left(\frac{1}{1+l+\mu}\right)^{M+N_{r}} \\
& \sum_{q=0}^{M}\left(\begin{array}{c}
M \\
q
\end{array}\right)\left(\frac{\mu}{1+\mu+l}\right)^{q} \int_{0}^{\nu} \frac{e^{-\frac{a(l+1)}{(\mu+l+1)}} a^{q+N_{r}-1}}{\left(N_{r}+q-1\right) !} d a \\
& =N_{t} \sum_{l=0}^{N_{t}-1} \sum_{\mathbf{b}} C_{l, \mathbf{b}}\left(\frac{1}{\mu+l+1}\right)^{M} \frac{\left(M+N_{r}-1\right) !}{\left(N_{r}-1\right) !} \\
& \sum_{k=0}^{M}\left(\begin{array}{c}
M \\
k
\end{array}\right)\left(\frac{\mu}{l+1}\right)^{k}\left(\frac{1}{l+1}\right)^{N_{r}} \gamma_{N_{r}+k}\left(\frac{\nu(l+1)}{\mu+l+1}\right) \text {. }
\end{aligned}
$$

Equation (18) is obtained using the relation $\int_{0}^{\infty} e^{-\alpha x} x^{n} d x=\frac{n !}{\alpha^{n+1}}$ when $n$ is an integer. The definition of $\gamma_{j+N_{r}}($.$) in integral form is used in equation (18) to get$ equation (19). Equation (20) is obtained by interchanging of the order of summation and integration. Equation (21) is obtained using the following simplification that uses the result in [32]:

$$
\begin{aligned}
& \sum_{j=0}^{\infty}\left(\frac{a \mu}{1+l+\mu}\right)^{j} \frac{\left(j+M+N_{r}-1\right) !}{j !\left(j+N_{r}-1\right) !} \\
& =e^{\left(\frac{a \mu}{1+\mu+l}\right)}\left(N_{r}+M-1\right) ! \sum_{q=0}^{M}\left(\begin{array}{c}
M \\
q
\end{array}\right) \frac{\left(\frac{a \mu}{1+\mu+l}\right)^{q}}{\left(N_{r}+q-1\right) !}
\end{aligned}
$$

Finally, equation (22) is obtained by re-writing the integral in terms of $\gamma_{N_{r}+k}($.$) .$

Therefore, we have obtained an upper bound for the outage probability of MIMO antenna selection system that is valid for all SNR. A lower bound can be obtained by evaluating the outage probability of MIMO antenna selection with perfect CSIR and feedback delay. In this case, we have an equality in equation (5). Therefore, the expression in equation (22) becomes an exact expression for outage probability in the perfect CSIR case with delayed CSIT. These results are numerically compared in the next section. Based on the results, it is clear that the upper bound derived above is close to the outage probability with perfect CSIR and delayed CSIT, i.e., the upper bound is tight.

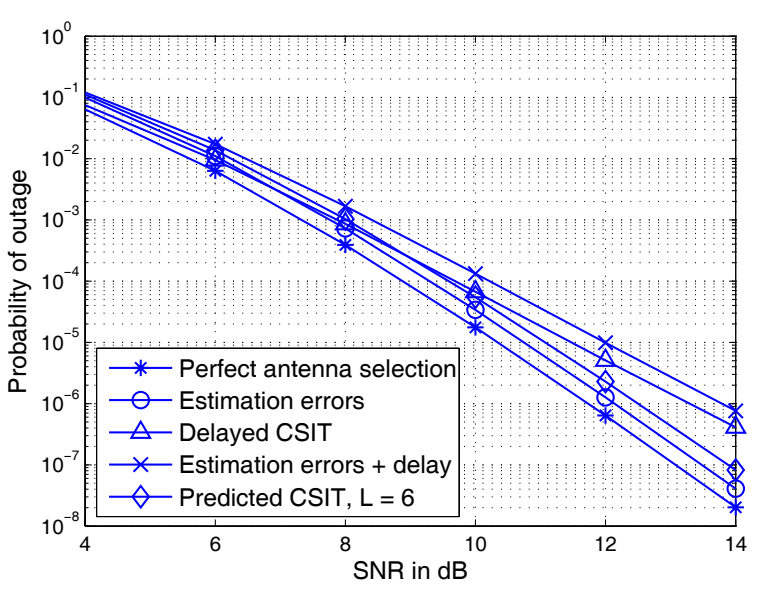

Fig. 3. Probability of outage vs. SNR for $2 \times 4$ system, normalized Doppler $=0.05$.

\section{A. Numerical Results}

The outage probability vs. SNR (average) for a $2 \times 4$ antenna selection system is depicted in Fig. 3. The average SNR is given by $S N R_{\text {average }}=\frac{P}{\sigma_{n}^{2}}=\frac{N_{t} P_{t}+\left(N-N_{t}\right) P_{d}}{N \sigma_{n}^{2}}$, where $P_{t}$ and $P_{d}$ are the transmit powers used during training and data respectively. The values of $P_{t}$ and $P_{d}$ can be optimized to improve the quality of the estimate. As in [22], the upper bound on outage probability in equation (6) is minimized if $P_{d}$ satisfies the equation $a P_{d}^{2}+b P_{d}+c=0$, where $a=\left(N-N_{t}\right)\left(N-2 N_{t}\right), b=-2\left(N-N_{t}\right)\left(N P+N_{t} \sigma_{n}^{2}\right)$ and $c=\left(N P+N_{t} \sigma_{n}^{2}\right)^{2}$. The desired rate $R$ is 2 nats $/ \mathrm{sec} / \mathrm{Hz}$. Normalized Doppler of $0.05\left(f_{d}=25 \mathrm{~Hz}, T=2 \mathrm{msec}\right)$ and delay $\Delta=1$ frame are considered, which lead to $J_{0}\left(2 \pi f_{d} T \Delta\right)=0.97$. The outage probability is plotted for the four imperfect CSI cases discussed in Section II-A and for the perfect CSIT case. It is evident from Fig. 3 that the degradation due to feedback delay becomes significant as SNR increases. While diversity order of 8 is achieved with perfect CSIT, feedback delay reduces the diversity order to 4 . On the other hand, estimation errors do not alter the diversity gain and lead to a fixed SNR loss. The upper bound on outage probability with feedback delay and estimation error is close to the outage probability with feedback delay and perfect CSIR. This shows that the upper bound is tight. Fig. 3 also reveals that performance degradation caused by delay can be significantly compensated using channel prediction.

The upper bound in equation (22) is validated using simulations. Fig. 4 compares the simulation results on the outage probability with the analytical results for the $2 \times 4$ system for $f_{d} T_{=} 0.05$ and $R=2$ nats $/ \mathrm{sec} / \mathrm{Hz}$. Results are shown for the imperfect CSIT case with estimation errors and feedback delay with and without prediction, corresponding to cases 3 and 4 in Section II-A. In both these cases, it can be seen that the upper bound is close to the simulation results, validating the analysis.

The outage probability is plotted vs. $R$ in Fig. 5 for a $2 \times 2$ system for SNR of $25 \mathrm{~dB}$. Normalized Doppler of 0.05 and $\Delta=2$ frames are considered. This plot can be used to determine the outage capacity for a given outage probability. It 


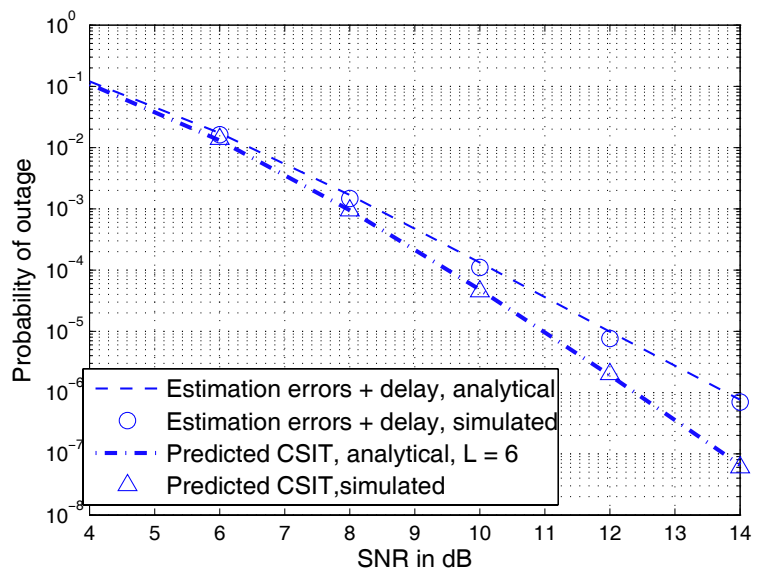

Fig. 4. Probability of outage vs. SNR for $2 \times 4$ system, normalized Doppler $=0.05$.

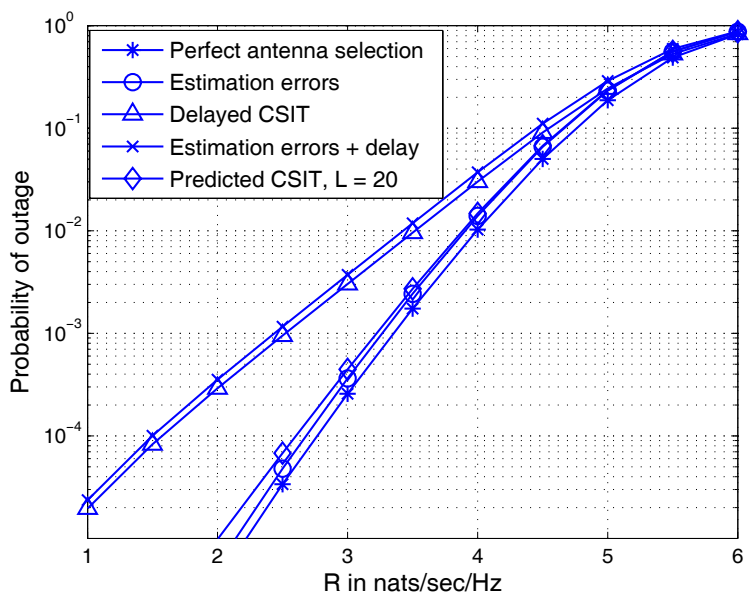

Fig. 5. Probability of outage vs. R for $2 \times 2$ system, normalized Doppler $=$ $0.05, \mathrm{SNR}=25 \mathrm{~dB}$.

can be seen that at outage probability of about $10^{-2}$, feedback delay leads to a degradation of about $12 \%$ in the achievable rate.

Fig. 6 shows the graph of outage capacity vs. SNR for $2 \times 2$ system for an outage probability of 0.01 . Normalized Doppler of 0.05 and $\Delta=2$ frames are considered. This plot again provides information on the achievable rate over the range of SNR for the given outage probability. There is a significant degradation in the achievable rate due to feedback delay (without channel prediction). In order to achieve the same rate, the required SNR is about $2 \mathrm{~dB}$ higher with feedback delay. This means that a rate-adaptive system needs to choose the transmission rate conservatively in the presence of feedback delay. However, prediction can be used to significantly compensate for the effect of delay.

\section{B. Required value for correlation}

In the previous subsection, we presented the outage probability of the MIMO antenna selection system with imperfect CSIT. It was evident that the effect of estimation errors can

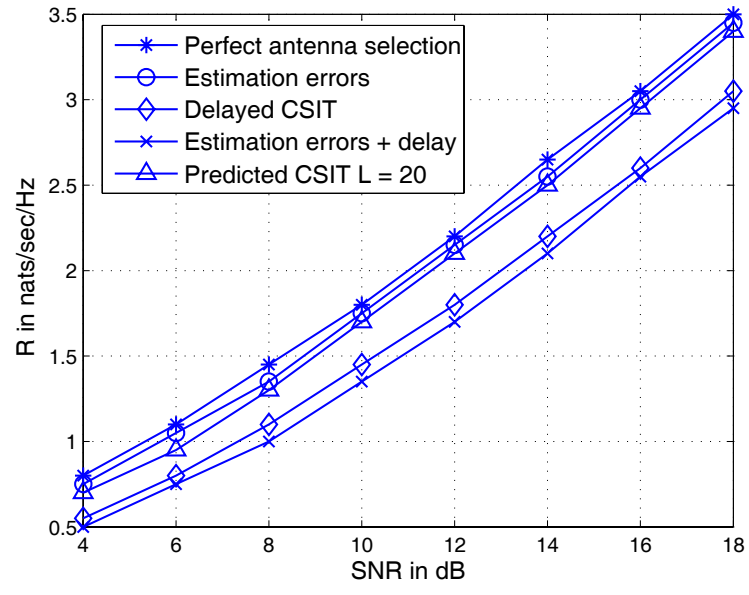

Fig. 6. Outage capacity vs. SNR for $2 \times 2$ system, normalized Doppler $=$ $0.05, \mathrm{P}($ outage $)=0.01$.

be characterized by a fixed SNR loss while feedback delay alters the diversity order. With constant $\rho$, the degradation in performance increases with increasing SNR. Therefore, the value of $\rho$ should increase with increasing SNR and approach 1 as $S N R \rightarrow \infty$ for the performance degradation to be bounded by a fixed loss in SNR. The minimum value of $\rho$, say $\rho_{t}$, required for the outage probability with imperfect CSIT to be bounded by $(1+\epsilon)$ times the outage probability without delay is calculated. The value of $\epsilon$ can be chosen based on the degradation in performance that is acceptable. The value of $\rho_{t}$ is calculated numerically and $1-\rho_{t}$ is plotted vs. SNR in Fig. 7 for a $2 \times 2$ system for $R=2$ nats $/ \mathrm{sec} / \mathrm{Hz}$. It can be observed that $1-\rho_{t} \propto S N R^{-1}$ at high SNR. Channel prediction using a finite length linear MMSE prediction filter can improve the value of $\rho$ and reduce the degradation for a larger range of SNR. However, at sufficiently high SNR, the degradation will be significant for any $\rho$ less than 1 . The value of $\rho$ obtained using prediction filters of length 2, 5, 10 and 100 for normalized Doppler of 0.05 and $\Delta=1$ frame are also plotted in the graph. It can be seen that prediction is not required up to a SNR of about $7 \mathrm{~dB}$. Filter length of 2 is sufficient till SNR $=12 \mathrm{~dB}$ and $L=10$ and 100 are sufficient to limit degradation at SNR of 25 and $40 \mathrm{~dB}$ respectively. The graph of the prediction filter length $L$ required to achieve $\rho_{t}$ is plotted in Fig. 8 for various normalized Doppler frequencies and $\Delta=1$ frame. The required $L$ increases with SNR and Doppler. It can be seen that at SNR of $30 \mathrm{~dB}, \mathrm{~L}=10,25$ and 100 are required for $f_{d} T=0.03,0.04$ and 0.05 respectively.

\section{Asymptotic Diversity ORder Analysis}

From equation (22) in section III, it is clear that in the presence of feedback delay, the outage probability is a weighted summation of gamma functions of different orders starting from $N_{r}$. This implies that the diversity order with feedback delay can be less than that with perfect CSIT (which is $N_{t} N_{r}$ ). The same was ascertained in the previous section, where it was shown that $\rho$ is required to improve with SNR to limit the degradation. In this section, we derive the diversitymultiplexing gain-feedback quality tradeoff for the MIMO 


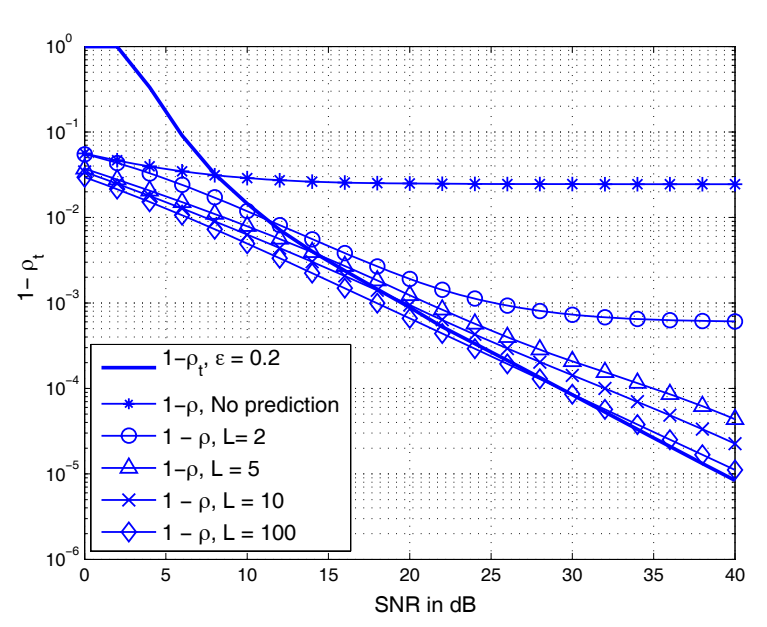

Fig. 7. $1-\rho_{t}$ vs. SNR for a $2 \times 2$ system, normalized Doppler $=0.05$.

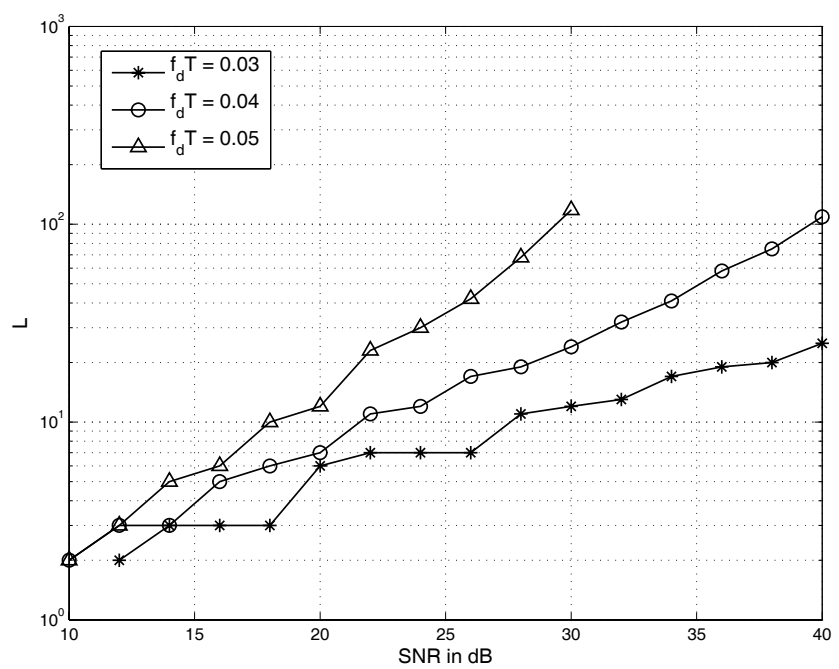

Fig. 8. $L$ vs. SNR for a $2 \times 2$ system.

antenna selection system with imperfect CSI. Feedback quality $\alpha$ is defined as the rate at which $\rho \rightarrow 1$ as $S N R \rightarrow \infty$, i.e.,

$$
\alpha=-\frac{\log (1-\rho)}{\log (S N R)} .
$$

The definition for the diversity gain $d$ and multiplexing gain $r$ are as given in [33]. With perfect CSIT, $d=N_{t} N_{r}(1-$ $r$ ). When $\alpha=0$, as in the delayed CSIT case, $\rho$ does not increase with SNR leading to a diversity gain of $N_{r}(1-r)$. The following theorem provides the diversity gain for other values of $\alpha$ and $r$ between 0 and 1 .

Theorem: The asymptotic diversity gain of the MIMO antenna selection system with imperfect CSIT is given by:

$$
d= \begin{cases}N_{r}\left(\alpha\left(N_{t}-1\right)+1-r\right) & \text { for } \alpha \leq 1-r \\ N_{t} N_{r}(1-r) & \text { for } \alpha>1-r,\end{cases}
$$

for $0 \leq \alpha \leq 1$ and $0 \leq r \leq 1$.

Proof: The proof follows a method similar to the method used in [34]. Consider equation (10). The region of integration over $Z$ can be split into two regions $[0, B]$ and $[B, \infty)$, where
$B$ is chosen such that $f_{Z}(z)=c \frac{\left(\frac{z}{\sigma_{t}^{2}}\right)^{K-1} e^{-\frac{z}{\sigma_{t}^{2}}}}{\sigma_{t}^{2}}+o\left(z^{K}\right)$ for $z \in[0, B]$ (for some constant $c$ ), and $\mathrm{K}^{\sigma_{t}^{2}}=N_{r} N_{t}$. Therefore, equation (10) can be written as [34]:

$$
P(\text { outage }) \leq T_{1}-T_{2}+T_{3},
$$

where

$$
\begin{aligned}
& T_{1}=c \int_{0}^{\infty} P\left(A_{1}<\nu / Z=Z_{1}=z\right) \frac{\left(\frac{z}{\sigma_{t}^{2}}\right)^{K-1} e^{-\frac{z}{\sigma_{t}^{2}}}}{\sigma_{t}^{2}} d z, \\
& T_{2}=c \int_{B}^{\infty} P\left(A_{1}<\nu / Z=Z_{1}=z\right) \frac{\left(\frac{z}{\sigma_{t}^{2}}\right)^{K-1} e^{-\frac{z}{\sigma_{t}^{2}}}}{\sigma_{t}^{2}} d z \\
& \text { and } T_{3}=\int_{B}^{\infty} P\left(A_{1}<\nu / Z=Z_{1}=z\right) f_{Z}(z) d z .
\end{aligned}
$$

Here $T_{1}-T_{2}$ corresponds to the integral over the region $[0, B]$ and $T_{3}$ corresponds to the integral over the other region.

\section{Analysis of Term $T_{1}$ :}

Using equation (12) in $T_{1}$ above, we have

$$
\begin{aligned}
T_{1} & =c \sum_{j=0}^{\infty} \gamma_{j+N_{r}}(\nu) \int_{0}^{\infty} \mu^{j} \frac{\left(\frac{z}{\sigma_{t}^{2}}\right)^{j+K-1} e^{-\frac{(\mu+1) z}{\sigma_{t}^{2}}}}{j ! \sigma_{t}^{2}} d z \\
& =\frac{c}{(1+\mu)^{K}} \sum_{j=0}^{\infty}\left(\frac{\mu}{1+\mu}\right)^{j} \frac{(j+K-1) !}{j !} \gamma_{j+N_{r}}(\nu) \\
& =\frac{c}{(1+\mu)^{K}} \sum_{j=0}^{\infty}\left(\frac{\mu}{1+\mu}\right)^{j} \frac{(j+K-1) !}{j !} \\
& \int_{0}^{\nu} \frac{e^{-a} a^{j+N_{r}-1}}{\left(j+N_{r}-1\right) !} d a \\
& =\frac{c}{(1+\mu)^{K}} \int_{0}^{\nu} e^{-a} a^{N_{r}-1} \sum_{j=0}^{\infty} \frac{(j+K-1) !}{\left(j+N_{r}-1\right) !} \frac{\left(\frac{\mu a}{1+\mu}\right)^{j}}{j !} d a \\
& =\frac{c}{(1+\mu)^{K}}(K-1) ! \sum_{p=0}^{K-N_{r}}\left(\begin{array}{c}
K-N_{r} \\
p
\end{array}\right) \frac{\left(\frac{\mu}{1+\mu}\right)^{p}}{\left(N_{r}+p-1\right) !} \\
& \int_{0}^{\nu} e^{-\frac{a}{1+\mu} a^{p+N_{r}-1} d a .}
\end{aligned}
$$

Equation (28) is obtained using the relation $\int_{0}^{\infty} e^{-\alpha x} x^{n} d x=\frac{n !}{\alpha^{n+1}}$ when $n$ is an integer. Using the definition of $\gamma_{j+N_{r}}$ (.) results in equation (29), while equation (30) is obtained by interchanging the order of summation and the integration. Equation (31) is obtained using the simplification similar to that in equation (23).

Replacing $a$ with $\frac{a}{1+\mu}$ and the integral with $\gamma_{p+N_{r}}($.$) , we$ get

$$
T_{1}=\frac{c}{(1+\mu)^{K-N_{r}}}(K-1) ! \sum_{p=0}^{K-N_{r}}\left(\begin{array}{c}
K-N_{r} \\
p
\end{array}\right) \mu^{p} \gamma_{p+N_{r}}\left(\frac{\beta}{\sigma_{r}^{2}}\right) .
$$

As $S N R \rightarrow \infty, \sigma_{r}^{2} \rightarrow 1, \beta \approx S N R^{-(1-r)}$ and $\mu \approx S N R^{\alpha}$. Note that $\gamma_{N}(x) \approx \frac{x^{N}}{N !}$ for small $x$. The terms of $T_{1}$, therefore 
decay with the rate $\alpha\left(K-N_{r}-p\right)+\left(p+N_{r}\right)(1-r), p=$ $0,1, \cdots,\left(K-N_{r}\right)$. Therefore, diversity gain

$$
d=\min _{p=0,1, \cdots, K-N_{r}} \alpha\left(K-N_{r}-p\right)+\left(p+N_{r}\right)(1-r) .
$$

When $\alpha<(1-r), p=0$ yields the minimum value of $d=N_{r}\left(\alpha\left(N_{t}-1\right)+(1-r)\right)$, while for $\alpha>1-r, p=K-N_{r}$ leads to minimum value of $d=N_{t} N_{r}(1-r)$. Therefore, the rate of decay of $T_{1}$ is $N_{r}\left(\alpha\left(N_{t}-1\right)+1-r\right)$ for $\alpha \leq(1-r)$ and $N_{t} N_{r}(1-r)$ for $\alpha>(1-r)$.

Analysis of Term $T_{2}$ :

$T_{2}$ can be written as

$$
\begin{aligned}
& T_{2}=c \sum_{j=0}^{\infty} \gamma_{j+N_{r}}(\nu) \int_{B}^{\infty} \mu^{j} \frac{\left(\frac{z}{\sigma_{t}^{2}}\right)^{j+K-1} e^{-\frac{(1+\mu) z}{\sigma_{t}^{2}}}}{\sigma_{t}^{2} j !} d z \\
& =\frac{c}{(1+\mu)^{K-1}} \sum_{j=0}^{\infty} \frac{\left(\frac{\mu}{1+\mu}\right)^{j}}{j !} \gamma_{j+N_{r}}(\nu) \frac{1}{\sigma_{t}^{2}} \\
& \int_{B}^{\infty}\left(\frac{(1+\mu) z}{\sigma_{t}^{2}}\right)^{j+K-1} e^{-\frac{(1+\mu) z}{\sigma_{t}^{2}}} d z \\
& =\frac{c}{(1+\mu)^{K}} \sum_{j=0}^{\infty} \frac{\left(\frac{\mu}{1+\mu}\right)^{j}}{j !} \gamma_{j+N_{r}}(\nu) \int_{\left(\frac{1+\mu}{\sigma_{t}^{2}}\right) B}^{\infty} e^{-x} x^{j+K-1} d x
\end{aligned}
$$

where equation (35) is obtained using $x=\frac{(1+\mu) z}{\sigma_{t}^{2}}$. At high SNR, $\nu \approx\left(\frac{1}{S N R}\right)^{1-r-\alpha}$.

When $\alpha \leq(1-r), \nu$ decreases with increasing SNR. Therefore, at high SNR, $\gamma_{j+N_{r}}(\nu) \approx \frac{\nu^{j+N_{r}}}{\left(j+N_{r}\right) !}$ and $T_{2}$ reduces to

$$
\begin{aligned}
T_{2} & \approx c\left(\frac{1}{1+\mu}\right)^{K-N_{r}}\left(\frac{\beta}{\sigma_{r}^{2}}\right)^{N_{r}} \\
& \sum_{j=0}^{\infty} \frac{\left(\frac{\mu \beta}{\sigma_{r}^{2}}\right)^{j}}{\left(j+N_{r}\right) ! j !} \int_{\left(\frac{1+\mu}{\sigma_{t}^{2}}\right) B}^{\infty} e^{-x} x^{j+K-1} d x .
\end{aligned}
$$

It is clear that the value of the integral tends to zero as $S N R \rightarrow \infty$. Therefore, for $\alpha \leq(1-r), T_{2}$ has terms that are $o\left(\alpha N_{r}\left(N_{t}-1\right)+(1-r) N_{r}\right)$, i.e., decay faster than $T_{1}$.

When $\alpha>(1-r), \nu$ increases with SNR. Therefore, at high SNR, $\gamma_{j+N_{r}}(\nu) \rightarrow 1$ and $T_{2}$ reduces to

$$
T_{2}=c\left(\frac{1}{1+\mu}\right)^{K} \sum_{j=0}^{\infty} \frac{\left(\frac{\mu}{1+\mu}\right)^{j}}{j !} \int_{\left(\frac{1+\mu}{\sigma_{t}^{\mu}}\right) B}^{\infty} e^{-x} x^{j+K-1} d x
$$

which is $o\left(\alpha N_{r} N_{t}\right)$.

Therefore, rate of decay of $T_{2}$ is higher than that of $T_{1}$ for all $\alpha, r$.

Analysis of Term $T_{3}$ :

Now consider $T_{3}$,

$$
T_{3}=\sum_{j=0}^{\infty} \gamma_{j+N_{r}}(\nu) \int_{B}^{\infty} \frac{e^{-\left(\frac{\mu z}{\sigma_{t}^{2}}\right)}\left(\frac{\mu z}{\sigma_{t}^{2}}\right)^{j}}{j !} f_{Z}(z) d z
$$

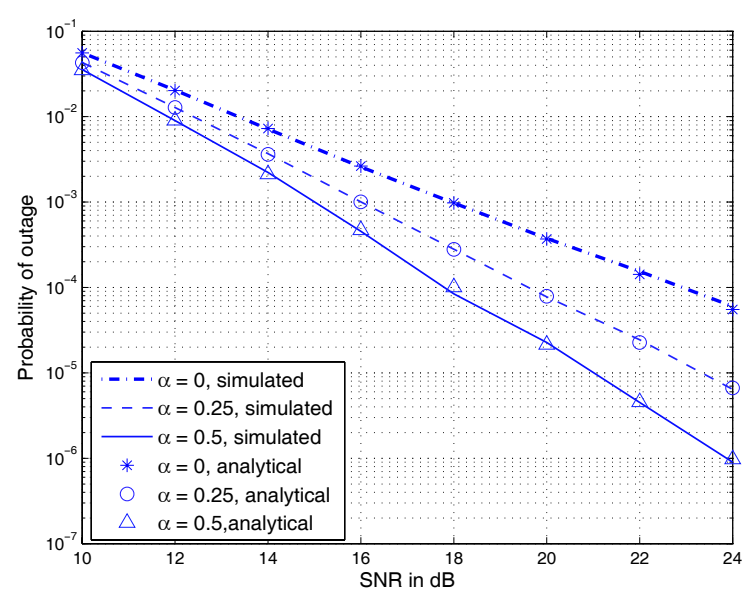

Fig. 9. Probability of outage vs. SNR $2 \times 2$ system, $\mathrm{R}=2$ nats $/ \mathrm{sec} / \mathrm{Hz}$.

But $\frac{e^{-\left(\frac{\mu z}{\sigma_{t}^{2}}\right)}\left(\frac{\mu z}{\sigma_{t}^{2}}\right)^{j}}{j !} \leq \Gamma_{j+1}\left(\frac{\mu z}{\sigma_{t}^{2}}\right)$, where $\Gamma_{j}(x)=e^{-x} \sum_{i=0}^{j-1} \frac{x^{i}}{i !}$ is the regularized upper incomplete gamma function. Since $\Gamma_{j}(x)$ is monotonically decreasing with $x$,

$$
\begin{aligned}
& T_{3} \leq \sum_{j=0}^{\infty} \gamma_{j+N_{r}}(\nu) \int_{B}^{\infty} \Gamma_{j+1}\left(\frac{\mu z}{\sigma_{t}^{2}}\right) f_{Z}(z) d z \\
& \leq \sum_{j=0}^{\infty} \gamma_{j+N_{r}}(\nu) \Gamma_{j+1}\left(\frac{\mu B}{\sigma_{t}^{2}}\right) \int_{B}^{\infty} f_{Z}(z) d z \\
& \leq \sum_{j=0}^{\infty} \gamma_{j+N_{r}}(\nu) \Gamma_{j+1}\left(\frac{\mu B}{\sigma_{t}^{2}}\right)
\end{aligned}
$$

which decays exponentially with increasing SNR.

Since $T_{2}$ and $T_{3}$ decay faster than $T_{1}$, the asymptotic diversity order is given by rate of decay of $T_{1}$. This results in the theorem.

The analytical asymptotic diversity order results are also compared with Monte Carlo simulations in Fig. 9 for a $2 \times 2$ system for $R=2$ nats $/ \mathrm{sec} / \mathrm{Hz}$. It can be seen that the diversity order is $2,2.5$ and 3 for $\alpha=0,0.25$, and 0.5 respectively.

\section{Conclusions}

The effect of feedback delay and channel estimation error on the performance of a MIMO antenna selection system have been analyzed. Feedback delay and channel estimation error affect the outage probability in different ways. Channel estimation error decreases with SNR and its effect is not significant at high SNR. However, feedback delay leads to significant degradation in outage probability as SNR increases. Furthermore, for rate adaptive systems, feedback delay leads to a significant reduction in the achievable rate for a given outage probability requirement. Channel prediction can be used to combat the effect of feedback delay. The calculation of the value of $\rho$ required to limit degradation revealed that the $1-\rho$ should be proportional to $S N R^{-1}$. This is later substantiated by the asymptotic diversity order analysis as a function of feedback quality. The length of the linear MMSE prediction filter required to achieve the desired $\rho$ is also calculated and 
found to increase with SNR. The asymptotic diversity order results show that as long as $1-\rho \propto S N R^{-1}$, the asymptotic diversity order is unaltered by imperfect CSIT.

\section{REFERENCES}

[1] E. Teletar, "Capacity of multi-antenna Gaussian channels," Eur. Trans. Telecomm., vol. 10, pp. 585-595, Nov. 1999.

[2] E. Biglieri, G. Caire, and G. Taricco, "Limiting performance of blockfading channels with multiple antennas," IEEE Trans. Inf. Theory, vol. 47, no. 4, pp. 1273-1289, May 2001.

[3] A. J. Goldsmith and P. P. Varaiya, "Capacity of fading channels with channel side information," IEEE Trans. Inf. Theory, vol. 43, no. 6, pp. 1986-1992, Nov. 1997.

[4] A. F. Molisch, M. Z. Win, and J. H. Winters, "Capacity of MIMO systems with antenna selection," in Proc. Int. Conf. Commun., Helsinki, Finland, vol. 2, pp. 570-574, June 2001.

[5] Z. Chen, J. Yuan, and B. Vucetic, "Analysis of transmit antenna selection/maximal ratio combining in Rayleigh fading channels," IEEE Trans. Veh. Technol., vol. 54, pp. 1312-1321, July 2005.

[6] D. A. Gore and A. Paulraj, "MIMO antenna subset selection with spacetime coding," IEEE Trans. Signal Process., pp. 2580-2588, vol. 50, no. 10, Oct. 2002.

[7] Z. Chen, "Analysis of maximal-ratio combining for generalized selection criterion," IEEE Commun. Lett., vol. 8, no. 4, pp. 247-249, Apr. 2004.

[8] A. F. Molisch, "MIMO systems with antenna selection-an overview," in Proc. IEEE Radio Wireless Conf., Boston, USA, pp. 167-170, Aug. 2003.

[9] R. Annavajjla and L. B. Milstein, "Performance analysis of linear diversity-combining schemes on Rayleigh fading channels with binary signaling and Gaussian weighting errors," IEEE Trans. Wireless Commun., pp. 2267-2278, vol. 4, no. 5, Sept. 2005.

[10] A. B. Narasimhamutrhy and C. Tepedelenlioglu, "Antenna selection for MIMO OFDM systems with channel estimation error," in Proc. IEEE Globecom 2007, pp. 3290-3294, Dec. 2007.

[11] W. M. Gifford, M. Z. Win, andM. Chiani, "Antenna Subset Diversity with Non-Ideal Channel Estimation," IEEE Trans. Wireless Commun., vol. 7, no. 5, pp. 1527-1529, May 2008.

[12] T. Gucluoglu, E. Panayirci, "Performance of Transmit and Receive antenna Selection in the Presence of Channel Estimation Errors," IEEE Commun. Letters, vol. 12, no. 5, pp. 371-373, May 2008.

[13] J. Tang, X. Zhang, Q. Du, "Alamouti scheme with joint antenna selection and power allocation over Rayleigh fading channels in wireless networks," in Proc. IEEE Globecom, pp. 3319-3323, Dec. 2005.

[14] D. V. P. Figueiredo, E. de Carvalho, L. Deneire, and R. Prasad, "Impact of feedback delay on rate adaptation for multiple antenna systems," in Proc. $17^{\text {th }}$ IEEE International Symp. Personal, Indoor Mobile Radio Communn., Sep. 2006.

[15] H. Sun, J. Wang. Y. Wang, and X. You, "Performance analysis of TASbased MISO system in temporally correlated channel," in Proc. $18^{\text {th }}$ IEEE International Symp. Personal, Indoor Mobile Radio Commun., Sep. 2007.

[16] S. Han and C. Yang, "Performance analysis of MRT and transmit antenna selection with feedback delay and channel estimation error," in Proc. IEEE Wireless Commun. Networking Conf., pp. 1135-1139, Mar. 2007.

[17] S. Zarrin and A. Gazor, "Analysis of partial phase combining, hybrid and transmit antenna selection schemes under channel estimation errors and feedback delay," in Proc. $10^{\text {th }}$ Canadian Workshop Inform. Theory, pp. 25-28, June 2007.

[18] V. S. Annapureddy, D. V. Marathe, T. R. Ramya, and S. Bhashyam, "Outage probability of multiple-input single-output (MISO) systems with delayed feedback," IEEE Trans. Commun., vol. 57, no. 2, pp. 319326, Feb. 2009.

[19] T. R. Ramya and S. Bhashyam, "Eigenbeamforming with delayed feedback and channel prediction," in Proc. IEEE ISIT 2009, Seoul, Korea, pp. 403-407, June-July 2009.
[20] Y. Li, N. B. Mehta, A. F. Molisch, and J. Zhang, "Optimal signaling and selection verification for single transmit-antenna selection," IEEE Trans. Commun., vol. 55, no. 4, pp. 778-789, Apr. 2007.

[21] B. Hassibi and B. M. Hochwald, "How much training is needed in multiple antenna wireless links?" IEEE Trans. Inf. Theory, vol. 49, no. 4, pp. 951-963, Apr. 2003

[22] S. Bhashyam, A. Sabharwal, and B. Aazhang, "Feedback gain in multiple antenna systems," IEEE Trans. Commun., vol. 50, no. 5, pp. 785-798, May 2002.

[23] S. Haykin, Adaptive Filter Theory. Prentice Hall Inc., 2002, 4th Edition.

[24] A. Papoulis, "A note on the predictability of band-limited processes," Proc. IEEE, pp. 1332, vol. 73, no. 8, Aug. 1985.

[25] A. Duel Hallen, and H. Hu, H. Hallen, "Long range prediction of fading signals," IEEE Signal Process. Mag., pp. 62-75, May 2000.

[26] A. Duel Hallen, "Fading channel prediction for mobile radio adaptive transmission systems," Proc. IEEE, vol. 95, no. 12, pp. 2299-2313, Dec. 2007.

[27] R. J. Lyman and A. Sikora, "Prediction of bandlimited fading envelopes with arbitrary spectral shape," IEEE Trans. Wireless Commun., vol. 6, no. 4, Apr. 2007

[28] L. Krasny, H. Arslan, D. Koilpillai, and S. Chennakeshu, "Doppler spread estimation in mobile radio systems," IEEE Commun. Lett., vol. 5 , no. 5, pp. 197-199, May 2001.

[29] J. Cai, W. Song, and Z. Li, "Doppler spread estimation for mobile OFDMsystems in Rayleigh fading channels," IEEE Trans. Consumer Electron., vol. 49, pp. 973-977, Nov. 2003.

[30] Y. Choi, O. C. Ozdural, H. Liu, and S. Alamouti, "A maximum likelihood doppler frequency estimator for OFDM systems," in Proc. IEEE International Conference on Communications 2006, pp. 45724576, vol. 10, June 2006.

[31] M. Abramowitz and I. A. Stegun, Handbook of Mathematical Functions. New York: Dover Publications, 1964, 9th printing.

[32] "Hypergeometric1F1Regularized." [Online]. Available: http://functions.wolfram.com/07.21.03.0022.01, Oct. 29, 2001 [Jun. 1, 2009].

[33] L. Zheng and D. N. C. Tse, "Diversity and multiplexing: a fundamental tradeoff in multiple-antenna channels," IEEE Trans. Inf. Theory, vol. 49, no. 5, pp. 1073-1096, May 2003.

[34] Z. Wang and G. B. Giannakis, "A simple and general parameterization quantifying performance in fading channels," IEEE Trans. Commun., vol. 51 , no. 8, Aug. 2003.

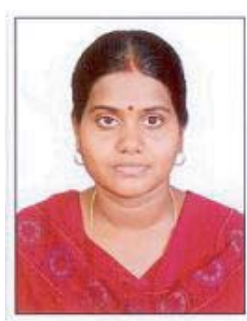

T. R. Ramya received the B.E. degree in Electronics and Communication Engineering from Anna University, Chennai, India in the year 1999 and the M.S. degree from Indian Institute of Technology Madras, India in the year 2002. From 2002-2004, she was a research associate at the HP-IITM R\&D Labs. She is currently pursuing her Ph.D at the Indian Institute of Technology Madras. Her interests include wireless communications and information theory.

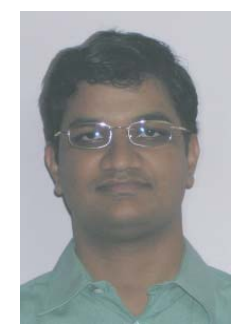

Srikrishna Bhashyam received his B.Tech. in Electronics and Communication Engineering from IIT Madras in 1996, and his M.S. and Ph.D. degrees in Electrical and Computer Engineering from Rice University, Houston, Texas, USA in 1998 and 2001 respectively. Between June 2001 and March 2003, he was a Senior Engineer at Qualcomm CDMA Technologies, Campbell, California, USA. Since May 2003, he has been with the Department of Electrical Engineering, IIT Madras, where he is currently Associate Professor. His current research interests are in resource allocation, adaptive transmission, code design, and information theory for multi-terminal wireless communication systems. He is currently on the editorial board of the IEEE TRANSACTIONS ON WIRELESS COMMUNICATIONS. 